\title{
Interleukin-18 expression and the response to treatment in patients with psoriasis
}

Hanaa Rasmy Mohamed Attia', Nancy Mikhael'2, Somaia Ismail ${ }^{3}$

${ }^{1}$ Clinical and Chemical Pathology Department, National Research Center, Cairo, Egypt 2Dermatology and Andrology Department, Benha Faculty of Medicine, Benha, Egypt ${ }^{3}$ Molecular Genetics Department, National Research Center, Cairo, Egypt

Submitted: 18 August 2009

Accepted: 22 February 2010

Arch Med Sci 2010; 6, 6: 964-970

DOI: 10.5114/aoms.2010.19309

Copyright @ 2010 Termedia \& Banach

\section{Abstract}

Introduction: The aim of the study was to demonstrate Interleukin-18 (IL-18) expression in keratinocytes from psoriatic lesions in comparison to keratinocytes from uninvolved skin and to study the change of expression after therapeutic interventions.

Material and methods: This study included 16 patients of different clinical subtypes of psoriasis. IL-18 gene expression analysis was performed using realtime quantitative PCR. Three biopsies were obtained from each patient. Two were taken from the lesional psoriatic skin and from uninvolved skin before starting treatment. A third lesional skin biopsy was taken at the end of two months' treatment course. The treatment was in the form of topical steroids or oral systemic methotrexate.

Results: Of all 16 studied patients significantly increased IL-18 expression was noted in keratinocytes from psoriatic lesions before and after treatment when compared to keratinocytes from uninvolved skin $(P=0.001$ and 0.002 respectively). The IL-18 expression in the skin lesions after treatment was significantly lower than lesional skin before treatment $(P=0.023)$. In psoriatic skin lesions of all studied patients IL-18 expression was significantly correlated with disease duration $(r=0.40$ and $P=0.01)$ and clinical severity of psoriasis $(r=0.72$ and $P=0.001)$.

Conclusions: Increased IL-18 expression in keratinocytes from psoriatic lesions of our patients and its correlation with disease duration and severity supported the concept which views psoriasis as a T-cell-mediated autoimmune disease. This could establish therapeutic and preventive approaches for psoriasis that ultimately lead to improved outcomes for patients.

Key words: psoriasis, interleukins, real-time PCR, effect of therapy

\section{Introduction}

Psoriasis is a chronic inflammatory condition which is the result of persistent stimulation of T cells by antigens of epidermal origin [1]. This persistent stimulation of $\mathrm{T}$ cells is the result of an interaction between T cells, antigen-presenting cells, i.e. Langerhans cells, and antigens, and comprises the following steps: primary signals (signal 1) including T-cell receptor stimulation by peptide antigen, co-stimulation (so-called accessory signals) (signal 2), and Thelper type 1 (Th 1) differentiation and proliferation [2].

In 1986, it was proposed that cytokines released by activated T-lymphocytes initiate and maintain the psoriatic process by stimulating keratinocyte proliferation [3]. Since that time it has become clear that, upon stimulation, keratinocytes are also able to secrete an array of different cytokines with a variety of functional effects on both themselves and other cell types, including T-lymphocytes [4].

\author{
Corresponding author: \\ Prof. Hanaa Rasmy Mohamed \\ Attia, MD, PhD \\ Clinical and Chemical \\ Pathology Department \\ Medical Division \\ National Research Center \\ Cairo, Egypt \\ 19 Ahmed Fahim, Nasr City \\ Cairo, Egypt \\ Phone: +2 0122208047 \\ E-mail: \\ Hanarasmy2000@yahoo.com
}


Interleukin-18 (IL-18) is related to the IL-1 family in terms of both structure and function. At the receptor level, the activity of IL-18 takes place through a signalling chain of a putative IL-18 receptor (IL-18R) complex [5].

Interleukin-18 was first described as an interferon- $\gamma$ (IFN- $\gamma$ ) inducing factor. In general, IL-18 induction of IFN- $\gamma$ is similar to that of IL-12, when it is a sole cytokine. IL-18 induces low levels of IFN$\gamma$, but in the presence of co-stimulants, IFN- $\gamma$ production is greatly enhanced [6]. However, because antibodies to IL-18 also reduced the hepatotoxicity of endotoxaemia, IL-18 was considered to possess other biological properties beyond that of inducing IFN- $\gamma$ [7]. IL-18 activates T cells to synthesize IL-2, GM-CSF, and TNF- $\alpha$. There are also reports that IL-18 suppresses the production of IL-10 and does not induce the production of IL$1 R a$. In general, the ability of IL-18 to induce different cytokines depends on the cellular targets [8]. Flisiak et al. confirmed an association between plasma IL18 concentration and psoriasis severity. Moreover, it was shown that combined measurement of IL-18 and TGF-beta1 in plasma can be considered as a possible biomarker of psoriasis activity [9]. Kato et al. indicated that a single nucleotide polymorphism (SNP) in the promoter of the interleukin-18 gene is associated with the presence of psoriasis, but not atopic dermatitis. This suggests that the SNP is associated with susceptibility to psoriasis vulgaris, presumably by affecting the production of IL-18 [10].

A few studies have analysed IL-18 expression in psoriasis vulgaris [11-14]. Much less information is available on the ability of different therapeutic interventions to modulate ongoing changes in expression in chronic disorders, especially in a human disease like psoriasis.

The aim of this study was to demonstrate expression of IL-18 in keratinocytes from psoriatic lesions in comparison to keratinocytes from nonlesional skin and to study the change of IL-18 expression after therapeutic interventions.

\section{Material and methods}

This study included 16 patients. The patients were selected from the outpatient clinic of Benha University Hospital, Al-Haud Al-Marsoud and The National Research Center, Cairo, Egypt. The patients included 4 (25\%) females and 12 (75\%) males, and their ages ranged from 21 to 62 years with a mean of $40.87 \pm 13.17$ years. They had different clinical subtypes of psoriasis and exhibited various degrees of severity. History of present illness included the onset, course, duration, previous treatment and date of last topical application or systemic therapy. Inclusion criteria: those included in the study were adult psoriatic patients $\geq 18$ years who should not have received a topical therapeutic modality apart from petrolatum for the last four weeks or systemic therapy for the last eight weeks.

Presence or absence of any coexisting noncutaneous conditions was established. Three patients were hypertensive, three had psoriatic arthropathy, one had hepatitis C virus (HCV) with anaemia of chronic disorder type and one had diabetes mellitus with hypertension. A positive family history was encountered only in one case. Two patients had upper respiratory tract infections; cold exposure worsened five patients' clinical presentation; sweating aggravated one patient's condition; three patients had psychogenic factors precipitating their cases. Only five of the studied patients were not affected by predisposing factors (31.3\%).

The psoriasis area and severity index (PASI) score sheet was adopted according to Fredriksson and Pettersson and Marks et al. $[15,16]$ for all patients as an indication of degree of severity. In mild cases, the extent of the disease does not exceed 10, while in severe cases, the extent of the disease is more than 30 based on the PASI score [17]. Informed consent was obtained after complete description of the study from each participant, according to the guidelines of the local ethical committee of the National Research Center.

Two biopsies were taken from each patient in the first set. The first biopsy was taken from the lesional psoriatic skin and the second from nonlesional skin. All patients were subjected to continuous treatment (in the form of topical steroids and oral systemic methotrexate) and followed up for 2 months. Six patients with moderate to severe degrees of psoriasis and two with palmoplantar subtype of psoriasis (who did not respond to topical steroids) received oral systemic methotrexate. The dosage used was: $15 \mathrm{mg}$ per week in three divided doses taken at 12hour intervals during a 24-hour period with folate supplementation. Patients receiving systemic methotrexate were subjected to monitoring liver blood tests monthly. Eight patients with mild to moderate degrees of psoriasis were maintained on topical steroid creams and ointments in the form of betamethasone dipropionate, twice daily. At the end of the course of two months, a third psoriatic lesional skin biopsy was taken from all patients.

\section{Tissue processing and preparation}

To prevent degradation by intracellular RNases, tissues were embedded soon after excision in RNA stabilizing reagent RNAlater provided from QIAGEN worldwide companies according to the manufacturer's instructions to be stored at $-70^{\circ} \mathrm{C}$ before sample processing. Frozen tissues were not allowed to thaw during handling to be ready for 
quantitative PCR determination of IL-18. Each biopsy was weighed before processing. Biopsies weighed up to $20 \mathrm{mg}$. Efficient disruption and homogenization of the starting material was performed using a rotor-stator homogenizer.

\section{RNA extraction and complementary (c) DNA synthesis}

Total RNA was extracted using QIA amp RNA extraction kit provided from QIAGEN worldwide companies. The concentration and purity of RNA were determined by measuring its absorbance at $260 \mathrm{~nm}\left(\mathrm{~A}_{260}\right)$ and $280 \mathrm{~nm}\left(\mathrm{~A}_{280}\right)$ in a UV visible spectrophotometer. Total RNA $(2 \mu \mathrm{g})$ was reversetranscribed to cDNA with High-Capacity cDNA Archive kit provided from Applied Biosystems. cDNAs were stored at $-20^{\circ} \mathrm{C}$ until real-time quantitative PCR was performed.

Table I. Demographic and behavioural characteristics of patients with psoriasis

\begin{tabular}{|c|c|}
\hline Characteristics & Patients \\
\hline Age range (mean $\pm \mathrm{SD}$ ) [years] & $21-62(40.875 \pm 13.17)$ \\
\hline $\operatorname{Sex}(M / F)$ & $12 / 4$ \\
\hline Marietal status (+/-) & $13 / 3$ \\
\hline Occupation (working/notworking) & $12 / 4$ \\
\hline Smoking (+/-) & $4 / 12$ \\
\hline $\begin{array}{l}\text { Duration of disease range } \\
\text { (mean } \pm \mathrm{SD} \text { ) in years }\end{array}$ & $\begin{array}{c}2 \text { months }-14 \text { years } \\
5.88 \pm 4.50\end{array}$ \\
\hline Family history (+/-) & $1 / 15$ \\
\hline Provoking factors (+/-) & $11 / 5$ \\
\hline Associated diseases (+/-) & $8 / 8$ \\
\hline
\end{tabular}

Table II. The range of the PASI score in the different types of psoriasis

\begin{tabular}{|lcccc|}
\hline Type of psoriasis & \multicolumn{4}{c|}{ PASI score grades } \\
\cline { 2 - 5 } & Mild* $^{*}$ & Moderate* & Severe $^{*}$ & Total (\%) \\
\hline Plaque-type & 3 & 2 & 1 & $6(37.5)$ \\
\hline Erythrodermic & & & 1 & $1(6.3)$ \\
\hline Guttate & & 1 & & $1(6.3)$ \\
\hline Flexural & 1 & & $1(6.3)$ \\
\hline Palmoplantar & 2 & & $2(12.5)$ \\
\hline Plaque and gutt & & 2 & $2(12.5)$ \\
\hline Plaque and p.p & 1 & & $1(6.3)$ \\
\hline Plaque and flex & & 1 & $1(6.3)$ \\
\hline Plaque and nails & & 1 & & $1(6.3)$ \\
\hline Total & 7 & 7 & 2 \\
\hline
\end{tabular}

gutt-guttate, p.p-plamoplantar, flex-flexural

*According to Ramsay and Lawrence [17]:

Mild cases - the PASI score did not exceed 10

Moderate cases - the PASI score was $>10$ to 30

Severe cases - the PASI score was more than 30

\section{Gene expression quantitation}

Real-time quantitative PCR was performed using Applied Biosystems Perkin Elmer 7300 sequence detection system. Primers were IL-18 sense primer 5'-AGG AAT AAA GAT GGC TGC TGA AC-3' and antisense primer 5'-GCT CAC CAC AAC CTC TAC CTC C3'. Each PCR reaction (in a $50 \mathrm{uL}$ volume) contained $1 \mathrm{x}$ of TaqMan universal PCR master mix, $(2 \mathrm{x}) 50$ to $900 \mathrm{nM}$ of forward primer, 50 to $900 \mathrm{nM}$ of reverse primer, $250 \mathrm{nM}$ of TaqMan probe labelled with 6-carboxyfluorescein (FAM) and 10 to $100 \mathrm{ng}$ of cDNA. The amplification protocol was $2 \mathrm{~min}$ at $50^{\circ} \mathrm{C}, 10 \mathrm{~min}$ at $95^{\circ} \mathrm{C}$, then 40 cycles for $15 \mathrm{sec}$ at $95^{\circ} \mathrm{C}$ and $1 \mathrm{~min}$ at $60^{\circ} \mathrm{C}$. Relative gene expression was determined for each sample. Amplification of the gene for glyceraldehydes-3-phosphate dehydrogenase (GAPDH), a constitutively expressed housekeeping gene, was performed on all samples. All genes were subsequently normalized against GAPDH levels. In this study mRNA levels were expressed in picograms/microlitre $(\mathrm{pg} / \mu \mathrm{l})$.

\section{Statistical analysis}

All data were collected from the patient charts and entered into a computerized spreadsheet. The fit of the data to the normal distribution was tested with the Kolmogorov-Smirnov test. Since the distribution of the data was significantly different from normal, nonparametric statistics were further used. Comparisons were made using MannWhitney U, Kruskal Wallis and Wilcoxon signed ranks tests between variables adjusted for appropriate covariates, and Spearman rank correlation coefficients were calculated between the assessed variables. The null hypothesis was rejected with a two-sided $P$ value of $<0.05$. All analyses were performed with SPSS 11.0 for Macintosh (Statistical Package for the Social Sciences. SPSS Inc., Chicago, IL, U.S.A.).

\section{Results}

Demographic and behavioural characteristics of included patients are presented in Table I. PASI score used to evaluate the severity of psoriasis varied from 1.4 to 39 with a mean of $14.96 \pm 11.82$. According to Ramsay and Lawrence [17], the PASI score grades in the different subtypes of psoriasis are demonstrated in Table II.

\section{Results of quantitative PCR}

All patients had detectable levels of IL-18 mRNA in the uninvolved skin. Table 3 shows the range, mean \pm SD and median levels of cytokine gene expression in each skin biopsy at the different times before and after receiving medication whether topical or systemic. Comparisons between median IL-18 expression levels with percentiles in all studied patients are presented in Figure 1. 
Table III. IL-18 expression levels in psoriatic patients

\begin{tabular}{|c|c|c|c|c|c|c|}
\hline \multirow[t]{2}{*}{ Variant } & \multirow[t]{2}{*}{$N$} & \multirow[t]{2}{*}{ Mean \pm SD } & \multirow[t]{2}{*}{ Range } & \multicolumn{3}{|c|}{ Percentiles } \\
\hline & & & & $25^{\text {th }}$ & $50^{\text {th }}$ (mMedian) & $75^{\text {th }}$ \\
\hline \multicolumn{7}{|c|}{ All studied patients } \\
\hline IL-18 NL & 16 & $1.10 \pm 2.14$ & $0.01-7.90$ & 0.0300 & $0.1850^{a}$ & 1.2675 \\
\hline IL-18 LB & 16 & $3.24 \pm 5.63$ & $0.17-22.70$ & 0.4900 & 0.9050 & 4.9000 \\
\hline IL-18 LA & 16 & $1.78 \pm 2.72$ & $0.01-8.10$ & 0.2750 & $0.4600^{b}$ & 1.5250 \\
\hline \multicolumn{7}{|c|}{ Patients on TS } \\
\hline IL-18 NL & 8 & $0.26 \pm 0.44$ & $0.01-1.3$ & 0.014 & $0.051^{c}$ & 0.337 \\
\hline IL-18 LB & 8 & $1.59 \pm 2.1$ & $0.17-6.4$ & 0.2275 & 0.53 & 2.53 \\
\hline IL-18 LA & 8 & $0.61 \pm .58$ & $0.012-1.6$ & 0.1275 & 0.415 & 1.2 \\
\hline \multicolumn{7}{|c|}{ Patients on MTX } \\
\hline IL-18 NL & 8 & $1.93 \pm 2.84$ & $0.012-7.9$ & 0.1625 & $0.685^{d}$ & 3.775 \\
\hline IL-18 LB & 8 & $4.89 \pm 7.56$ & $0.49-22.7$ & 0.602 & 1.385 & 6.2 \\
\hline IL-18 LA & 8 & $2.95 \pm 3.52$ & $0.26-8.10$ & 0.3275 & 0.745 & 7.26 \\
\hline
\end{tabular}

IL-18 NL-IL-18 in non-lesional skin, IL-18 LB - IL-18 in lesional skin before treatment, IL-18 LA - IL-18 in lesional skin after treatment, TS - topical steroids, MTX - methotrexate

asignificantly lower than lesional skin before treatment $(P=0.001)$ and lesional skin after treatment $(P=0.002)$

${ }^{b}$ Significantly lower than lesional skin before treatment $(P=0.023)$

cSignificantly lower than lesional skin before treatment $(P=0.012)$ and after treatment $(P=0.012)$

dSignificantly lower than lesional skin before treatment $(P=0.018)$

Significantly lower IL-18 expression in uninvolved skin than lesional skin before treatment $(P=0.001)$ and lesional skin after treatment $(P=0.002)$ was observed in all studied patients as one group. The IL-18 expression in the psoriatic skin lesions after treatment was significantly lower than lesional skin before treatment $(P=0.023)$.

IL-18 mean expression levels in non-lesional and lesional skin (before and after treatment) in the

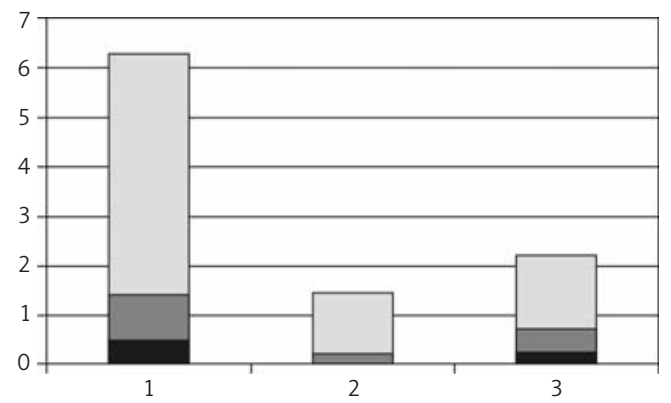

1 - IL-18 in lesional skin before treatment

2 - IL-18 in non lesional skin

3-IL-18 lesional skin after treatment

$\square 75-100^{\text {th }}$ percentile $\square 25-75^{\text {th }}$ percentile $\square 0-25^{\text {th }}$ percentile

Figure 1. Comparisons between median IL-18 expression levels with percentiles in all studied patients. Significantly lower IL-18 expression in uninvolved skin than lesional skin before treatment $(P=0.001)$ and lesional skin after treatment $(P=0.002)$ was demonstrated in all studied patients as one group. The IL-18 expression in the psoriatic skin lesions after treatment was significantly lower than lesional skin before treatment $(P=0.023)$ different subtypes of psoriasis are shown in Figure 2 . The highest expression level was noted in plaque and nails clinical subtype of psoriasis.

Among patients receiving topical steroids significantly lower IL-18 expression was detected in non-lesional skin than lesional skin before treatment $(P=0.012)$ and after treatment $(P=0.012)$. No statistical significance was obtained when comparing the change of expression before and after topical treatment $(P=0.069)$.

Individual variations of the IL-18 expression levels in non-lesional and lesional skin before and

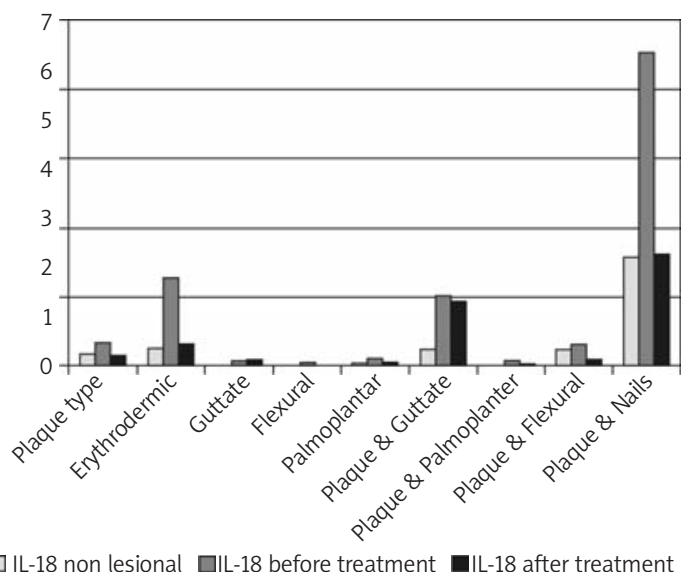

Figure 2. IL-18 expression levels in non lesional and lesional skin (before and after treatment) in the different subtypes of psoriasis. The highest expression level was noted in plaque and nails clinical subtype of psoriasis 


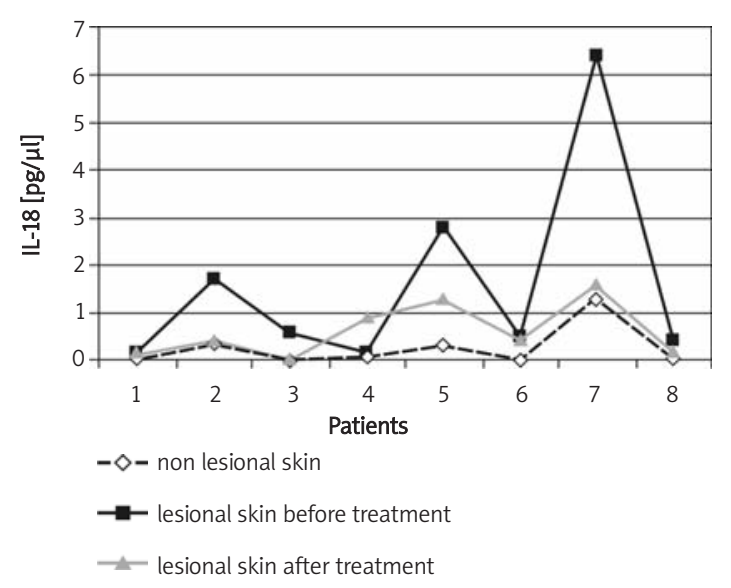

Figure 3. The individual variations of the IL-18 expression levels in non lesional and lesional skin before and after receiving topical steroids. Significantly lower IL-18 expression was detected in non lesional skin than lesional skin before treatment $(P=0.012)$ and after treatment $(P=0.012)$. No statistical significance could be obtained when comparing the change of expression before and after topical treatment $(P=0.069)$

after receiving topical steroids are presented in Figure 3.

In patients receiving methotrexate, significantly lower IL-18 expression was detected in non-lesional skin than lesional skin before treatment $(P=0.018)$. The change of expression levels in lesional skin after treatment was not statistically significant when compared to nonlesional skin $(P=0.069)$ and lesional skin before treatment $(P=0.12)$.

Individual variations of the IL-18 expression levels in non-lesional and lesional skin before and after receiving systemic methotrexate are shown in Figure 4.

In the correlation between IL-18 expression and different demographic and behavioural characteristics of the studied patients as one group, a significant correlation was obtained between IL-18 expression levels in psoriatic lesions and the severity of psoriasis expressed by PASI score $(r=0.72$ and $P=0.001)$ and disease duration $(r=0.40$ and $P=0.01)$. No significant correlation was revealed between IL-18 expression levels in psoriatic lesions and patients' age, smoking status, predisposing factors or coexisting diseases.

\section{Discussion}

In the present study our patients with psoriasis showed significantly increased IL-18 expression in keratinocytes from psoriatic lesions before and after receiving medication whether topical or systemic in comparison to keratinocytes from non-lesional skin. This supports the concept viewing psoriasis as a T-cell-mediated autoimmune disease. Krueger

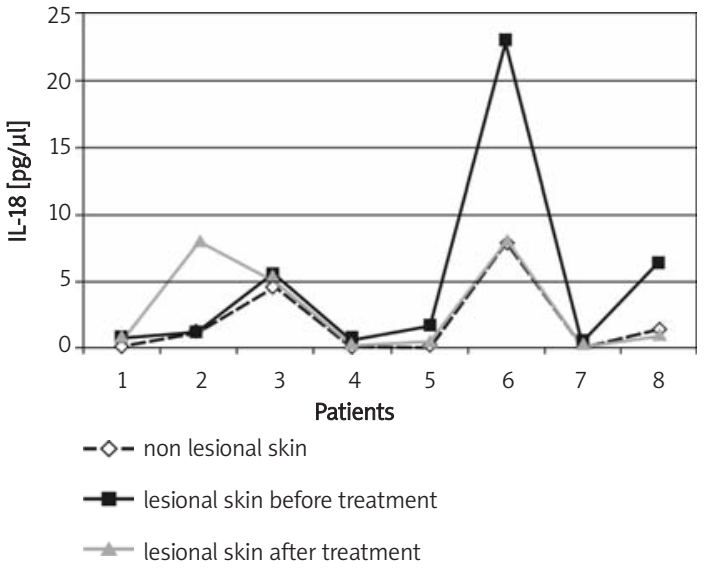

Figure 4. The individual variations of the IL-18 expression levels in non lesional and lesional skin before and after receiving systemic methotrexate. significantly lower IL-18 expression was detected in non lesional skin than lesional skin before treatment $(P=0.018)$. The change of expression levels in lesional skin after treatment was not statistically significant when compared to non lesional skin $(P=0.069)$ and lesional skin before treatment $(P=0.12)$

[18] reported, in his study on a large series of skin biopsies, increased protein expression in lesional as opposed to non-lesional skin samples. Other investigators stated that lesional skin might be the source of the elevated plasma levels of IL-18 [19]. In the present work quantitative PCR using mRNA taken from uninvolved skin revealed low but detectable levels of IL-18 mRNA expression. This finding is consistent with previous studies showing that human keratinocytes are capable of synthesizing low levels of IL-18 mRNA even under non-stimulated conditions [20]. Chang et al. [21] found that genes specifically modulated in uninvolved skin play a major role in triggering disease progression, and in being the first candidate for early disease diagnosis or development of new therapies. This finding could allow selection of the treatment regimen for individual patients.

In the present study the IL-18 expression in patients receiving topical steroids was significantly lower in non-lesional skin than lesional skin before and after treatment. After 2 months of treatment, IL-18 expression levels returned back to low values but not significantly lower than the lesional skin levels of expression before treatment. In patients receiving methotrexate non-lesional skin expression was significantly lower than lesional skin before treatment. Also the expression levels decreased after treatment but still not statistically significantly when compared to lesional skin before treatment. Otkjaer et al. [22] determined IL-19 and IL-20 in nonlesional and lesional psoriatic skin after 28 days of treatment using topical calcipotriol ointment for mild cases and oral cyclosporine for moderate and severe cases. They found that in patients treated 
with calcipotriol, IL-19 and IL-20 mRNA expression levels in lesional psoriatic skin were reduced after 14 days, but the reduction became statistically significant only after 28 days. So the timing for mRNA expression analysis could affect the degree of significance. This is further supported by studies demonstrating a decrease of IL-18 levels after narrowband UVB therapy together with other parameters' characteristics for the T helper 1 (Th1) response [23]. Apart from stimulating the Th1 response, IL-18 can regulate the Th2 response depending on the local cytokine network. IL-12 enhances IFN- $\gamma$ production induced by IL-18, whereas IL-18 alone induces IL-4 and IL-13 production [24].

Classic systemic treatments for psoriasis have not fully met the needs of patients for permanent improvement. Antibody-based or fusion proteinbased selective targeting of key mediators of inflammation has been added to the treatment approaches for psoriasis. Kong et al., and Smeltz $[25,26]$ studied IL-18 receptor blocking by using 1,25-dihydroxyvitamin D in mouse keratinocytes. They observed that the IL-18 expression level was decreased synchronously with treatment. Tak et al. [27] investigated IL-18 binding protein (IL-18 BP) in psoriatic patients and demonstrated that IL-18 BP neutralizes the activity of IL-18.

Our study demonstrated that IL-18 expression in the psoriatic lesions before treatment was significantly correlated with the severity of psoriasis and disease duration. No correlation was noted between IL-18 expression and patients' age, smoking status, predisposing factors or coexisting diseases. Flisiak et al. [9] found a significant correlation between plasma IL-18 levels and PASI values. But they did not find any correlation between IL-18 concentration in scales and PASI score. Trinquet et al. [28] studied the influence of disease duration on gene expression profiles in psoriasis. They did not reveal any correlation. However, Craven et al. [29] studied IL-10 in early and late onset psoriasis and confirmed that IL-10 expression was correlated significantly with disease duration. Sampogna et al. [30] studied 380 patients with psoriasis and did not find any significant difference between older and younger patients regarding IL-18 levels. On the other hand, Chen et al. [31] found a statistically significant correlation between IL-18 expression levels and age, with a $P$ value of 0.015

In conclusion, based on the increased IL-18 expression levels in psoriatic skin lesions relative to uninvolved skin, this cytokine appears to be crucial in the development of the active psoriatic lesion itself, where it is produced locally by a step in the evolution of the psoriatic lesion. This could be supported by its correlation with clinical severity and disease duration, and reduction of its expression after treatment. The decreased expression after treatment was not to an extent indicating that the skin had returned to normal. The small size of the study population and the heterogeneous group of patients participating in the study preclude the drawing of firm conclusions. Future studies on genes involved and array-based technologies should allow confirmation of the results obtained. Also further studies are recommended to try other lines of treatment for psoriasis. One of these lines of therapy is to try $\mathrm{r}$-h IL-18 BP to neutralize IL-18 activity on a wide scale that will ultimately lead to improved outcomes for patients.

\section{References}

1. Homey B, Dieu-Nosjean, MC, Wiesenborn A, et al. Upregulation of macrophage inflammatory protein-3 alpha/CCL20 and CC chemokine receptor 6 in psoriasis. J Immunol 2000; 164: 6621-32.

2. Krueger JG. The immunologic basis for the treatment of psoriasis with new biologic agents. J Am Acad Dermatol 2002; 46: 1-23.

3. Valdimarsson H, Baker BS, Jonsdottir I, Fry L. Psoriasis: a disease of abnormal keratinocyte proliferation induced by T-lymphocytes. Immunol Today 1986; 7: 256-9.

4. Barker BS, Fry L. The immunology of psoriasis. Br J Dermatol 1992; 126: 1-9.

5. Torigoe K, Ushio S, Okura T, Kobayashi S, Taniai M, Kunikate T. Purification and characterization of the human interleukin-18 receptors. J Biol Chem 1997; 272: 25737-42.

6. Mastroeni P. Immunity to systemic salmonella infections. Curr Mol Med 2002; 2: 393-406.

7. Dinarello CA. Novel target for interleukin-18 binding protein. Ann Rheum Dis 2001; 60: 18-24.

8. Wigginton JM, Wiltrout RH. IL-12, IL-2 combination cytokine therapy for solid tumours: translation from bench to beside. Expert. Opin Biol Ther 2002; 2: 513-24.

9. Flisiak I, Klepacki A, Chodynicka B. Plasma and scales levels of interleukin 18 in comparison with other possible clinical and laboratory biomarkers of psoriasis activity. Biomarkers 2006; 11: 194-200.

10. Kato T, Tsunemi Y, Saeki H, et al. Interferon-18 gene polymorphism $-137 \mathrm{G} / \mathrm{C}$ is associated with susceptibility to psoriasis vulgaris but not with atopic dermatitis in Japanese patients. J Dermatol Sci 2009; 53: 162-3.

11. McKenzie RC, Boyce F, Forsey R, et al. Psoriatic skin expresses high levels of interleukin-18 (IL-18) and IL-18 receptor (IL-18R). Br J Dermatol 2000; 142: 618.

12. Ohta Y, Hamada Y, Katsuoka K. Expression of IL-18 in psoriasis. Arch Dermatol Res 2001; 293: 334-42.

13. McKenzie RC, Boyce F, Szepietowski JC, et al. Psoriatic epidermis expresses high levels of interleukin 18 (IL-18), IL-18 receptor mRNA and IL-18. Dermatol Klin 2002; 1-4: $17-23$.

14. McKenzie RC, Sabin E, Szepietowski JC, Gracie JA, Forsy RJ, Howie S. Interferon gamma in keratinocytes in psoriasis. Eur J Dermatol 2003; 13: 315-6.

15. Fredriksson T, Pettersson U. Severe psoriasis-oral therapy with a new retinoid. Dermatologica 1978; 157: 238-44.

16. Marks R, Barton S, Shuttelworth D, Finlay AY. Assessment of disease progress in psoriasis. Arch Dermatol 1989; 125: 235-40.

17. Ramsay B, Lawrence CM. Measurement of involved surface area in patients with psoriasis. Br I Dermatol 1991; 124: 565-70. 
18. Krueger J. What clinical trials teach us about the biology of psoriasis? Br J Dermatol 2006; 154: 461-6.

19. Yamanaka K, Clark R, Dowgiert R, et al. Expression of interleukin-18 and caspase-1 in cutaneous T-cell lymphoma. Clin Cancer Res 2006; 12: 376-82.

20. Koizumi H, Sato-Matsumura KC, Nakamura $\mathrm{H}$, et al. Distribution of IL-18 and IL-18 receptor in human skin: various forms of IL-18 are produced in keratinocytes. Arch Dermatol Res 2001; 293: 325-33.

21. Chang C, Magracheva E, Kozlov S, et al. Crystal structure of interleukin-19 defines subfamily of helical cytokines. J Biol Chem 2003; 278: 3308-13.

22. Otkjaer K, Kragballe KV, Funding AT, et al. Interleukin 19 and interleukin-20 and their receptors in psoriasis. $\mathrm{Br}$ J Dermatol 2005; 153: 911-8.

23. Piskin G, Tursen U, Sylva-Steenl RMR, Bos JD, Teunissen MBM. Clinical improvement in chronic plaque type psoriasis lesion after narrow band UVB therapy is accompanied by a decrease in the expression of IFN- $\gamma$ inducers IL-12 IL-18 and IL-23. Exp Dermatol 2004; 13: 764-72.

24. Nakanishi K, Yoshimoto T, Tsutsui H, Okamura H. Interleukin-18 regulates both Th1 and Th2 responses. Annu Rev Immunol 2001; 19: 423-74.

25. Kong J, Grando SA, Li YC. Regulation of IL-1 family cytokines IL-1 $\alpha$, IL-1 receptor antagonist, and IL-18 by 1,25dihydroxyvitamin D in primary keratinocytes. J Immunol 2006; 176: 3780-7.

26. Smeltz RB. Profound enhancement of IL-12/IL-18 pathway of IFN- $\gamma$ section in human $C D 8^{+}$memory $T$ cell subset via IL-15. J Immunol 2007; 178: 4786-92.

27. Tak PP, Bacchi M, Bertolino M, Desson A. Pharmacokinetics of IL-18 binding protein in healthy volunteers and subjects with rheumatoid arthritis or plaque psoriasis. Eur J Drug Metab Pharmacokinet 2006; 31: 109-16.

28. Trinquet QV, Fogel P, Aldana-Jammayrac $O$, et al. Interleukin 19 gene expression profile in psoriasis: anaylsis of impact of body site location and clinical severity. Br J Dermatol 2005; 152: 489-504.

29. Craven NM, Jackson CW, Kirby B, et al. Cytokine gene polymorphism in psoriasis. Br J Dermatol 2001; 144: 849-53.

30. Sampogna F, Tabolli S, Söderfeldt B, et al. Measuring quality of life of patients with different clinical types of psoriasis using the SF-36. Br J Dermatol 2006; 154: 844-9.

31. Chen WJ, Yang JY, Lin JH, et al. Nasopharyngeal shedding of severe acute respiratory syndrome associated corona virus is associated with genetic polymorphism. Clin Infect Dis 2006; 42: 1561-9. 